\title{
Diagnosing and Managing Postherpetic Neuralgia
}

\author{
Srinivas Nalamachu $\cdot$ Patricia Morley-Forster
}

Published online: 5 October 2012

(C) The Author(s) 2012. This article is published with open access at Springerlink.com

\begin{abstract}
Postherpetic neuralgia (PHN) represents a potentially debilitating and often undertreated form of neuropathic pain that disproportionately affects vulnerable populations, including the elderly and the immunocompromised. Varicella zoster infection is almost universally prevalent, making prevention of acute herpes zoster (AHZ) infection and prompt diagnosis and aggressive management of PHN of critical importance. Despite the recent development of a herpes zoster vaccine, prevention of AHZ is not yet widespread or discussed in PHN treatment guidelines. Diagnosis of PHN requires consideration of recognized PHN signs and known risk factors, including advanced age, severe prodromal pain, severe rash, and AHZ location on the trigeminal dermatomes or brachial plexus. PHN pain is typically localized, unilateral and chronic, but may be constant, intermittent, spontaneous and/or evoked. PHN is likely to interfere with sleep and daily activities. First-line therapies for PHN include tricyclic antidepressants, gabapentin and pregabalin, and the lidocaine $5 \%$ patch. Second-line therapies include strong and weak opioids and topical capsaicin cream or $8 \%$ patch. Tricyclic antidepressants, gabapentinoids and strong opioids are effective but are also associated with systemic adverse events that may limit their use in many patients, most notably those with significant medical comorbidities
\end{abstract}

S. Nalamachu $(\bowtie)$

International Clinical Research Institute, Inc., 8675 College

Blvd, Suite 150, Overland Park, KS 66210, USA

e-mail: nalamachu@yahoo.com

\section{P. Morley-Forster}

Interdisciplinary Pain Program, University of Western Ontario, St. Josephs Hospital, Room B3-628, 268 Grosvenor Street, London, ON N6A 4V2, Canada

e-mail: pat.morley-forster@sjhc.london.on.ca or advanced age. Of the topical therapies, the topical lidocaine $5 \%$ patch has proven more effective than capsaicin cream or $8 \%$ patch and has a more rapid onset of action than the other first-line therapies or capsaicin. Given the low systemic drug exposure, adverse events with topical therapies are generally limited to application-site reactions, which are typically mild and transient with lidocaine $5 \%$ patch, but may involve treatment-limiting discomfort with capsaicin cream or $8 \%$ patch. Based on available clinical data, clinicians should consider administering the herpes zoster vaccine to all patients aged 60 years and older. Clinicians treating patients with PHN may consider a trial of lidocaine $5 \%$ patch monotherapy before resorting to a systemic therapy, or alternatively, may consider administering the lidocaine $5 \%$ patch in combination with a tricyclic antidepressant or a gabapentinoid to provide more rapid analgesic response and lower the dose requirement of systemic therapies.

\section{Introduction}

Reactivation of a dormant varicella zoster virus, the virus that causes chicken pox, yields acute herpes zoster (AHZ) or shingles, a painful blistering skin rash $[1,2]$. More than $95 \%$ of the adult population in developed countries manifest seropositivity for antibodies to the varicella zoster virus, and are, thus, at risk for developing AHZ [1]. After the initial varicella zoster infection, the virus persists in the ganglia of the sensory cranial nerves and spinal dorsal root ganglia without triggering symptoms for many years [2]. However, with age-, stress- or illness-related diminution in cell-mediated immunity, the risk for AHZ increases. Indeed, AHZ affects approximately $33 \%$ of the population and generally, the risk increases dramatically with age [1-3]. 
The Olmsted County Study found that the incidence of AHZ was 3.6 cases per 1,000 person-years, but was low in individuals aged 20-29 years and spiked to 7.1 cases per 1,000 person-years in the seventh decade of life and reached 12.0 cases per 1,000 person years in individuals aged $\geq 80$ years. In all, $51 \%$ of AHZ infection cases occurred in individuals aged $\geq 60$ years, although this age group represents only $23 \%$ of the US population [4].

Although the symptoms of AHZ typically resolve within 2-4 weeks, approximately $10 \%$ of patients develop postherpetic neuralgia (PHN), often defined as pain persisting more than 3 months after the onset of the rash in the same affected area (Fig. 1) [3, 5]. The risk of PHN increases with age. In the Olmsted County Study, $73 \%$ of PHN cases were in individuals aged $\geq 60$ years [4]. PHN pain results from sensory nerve damage and may be intermittent, chronic or spontaneous in nature [2]. PHN symptoms frequently include allodynia, wherein pain is evoked by normally nonpainful mechanical stimuli, such as light brushing of the skin. Even with appropriate treatment, PHN pain can interfere with sleep and routine daily activities.

Despite the potentially debilitating nature of this disorder, PHN tends to be underdiagnosed and inadequately managed, especially in primary care. Several factors may contribute to the undertreatment of PHN. When a patient reports chronic pain, clinicians may not always follow up by taking a complete patient history or performing a thorough physical examination to identify the source of the pain, steps particularly important in elderly and medically complicated patients who are at high risk of PHN. Additionally, clinicians may not be familiar with the symptoms of PHN and the most effective available treatment options. This review provides essential information that will enable clinicians to correctly diagnosis PHN and select the most appropriate pharmacotherapy for their patients with PHN.

\section{Prevention of Acute Herpes Zoster}

A herpes zoster vaccine $\left(\operatorname{Zostavax}^{\circledR}\right)$ received US FDA approval in 2006 for use in adults aged 60 years or older [6] and in 2011 was approved for use in those aged $\geq 50$ years [7]. In the Shingles Prevention Study, a large (>38,000 subjects), 3-year trial in older adults ( $\geq 60$ years), subjects were randomized to treatment with either the herpes zoster vaccine or placebo [8]. The vaccine reduced the incidence of herpes zoster infection versus placebo by approximately $50 \%$ (315 cases vs. 642 cases). A total of 107 subjects experienced PHN. The herpes zoster vaccine was shown to significantly reduce the incidence of PHN versus placebo by approximately two-thirds (27 cases vs. 80 cases; $p<0.001)$. Greater reductions in $\mathrm{PHN}$ than in $\mathrm{AHZ}$ suggest that vaccinated patients who develop AHZ are less likely to progress to PHN.

In the Shingles Prevention Study, other investigators have shown that the herpes zoster vaccine was well tolerated, with the incidence of serious adverse events similar to that seen with placebo [9]. However, this study excluded subjects with prior herpes zoster infection younger than 60 years and immunocompromised individuals, and it was conducted only in the USA. As a consequence, the findings cannot be generalized to all populations at risk for PHN [6]. A subsequent study evaluating $>22,000$ individuals aged 50-59 years in the USA and Europe found that the vaccination reduced the incidence of AHZ by approximately $70 \%$, although the study period was only 1 year [10].

In addition, a vaccine cost-effectiveness analysis based on the Shingles Prevention Study demonstrated that the benefits of the vaccine were not evenly distributed [11]. Although the vaccine was most effective in younger
Fig. 1 Left image shows typical presentation of acute herpes zoster. Right image illustrates typical presentation of postherpetic neuralgia, with pain persisting $>4$ months after the acute rash, which may have completely disappeared or left scant scarring
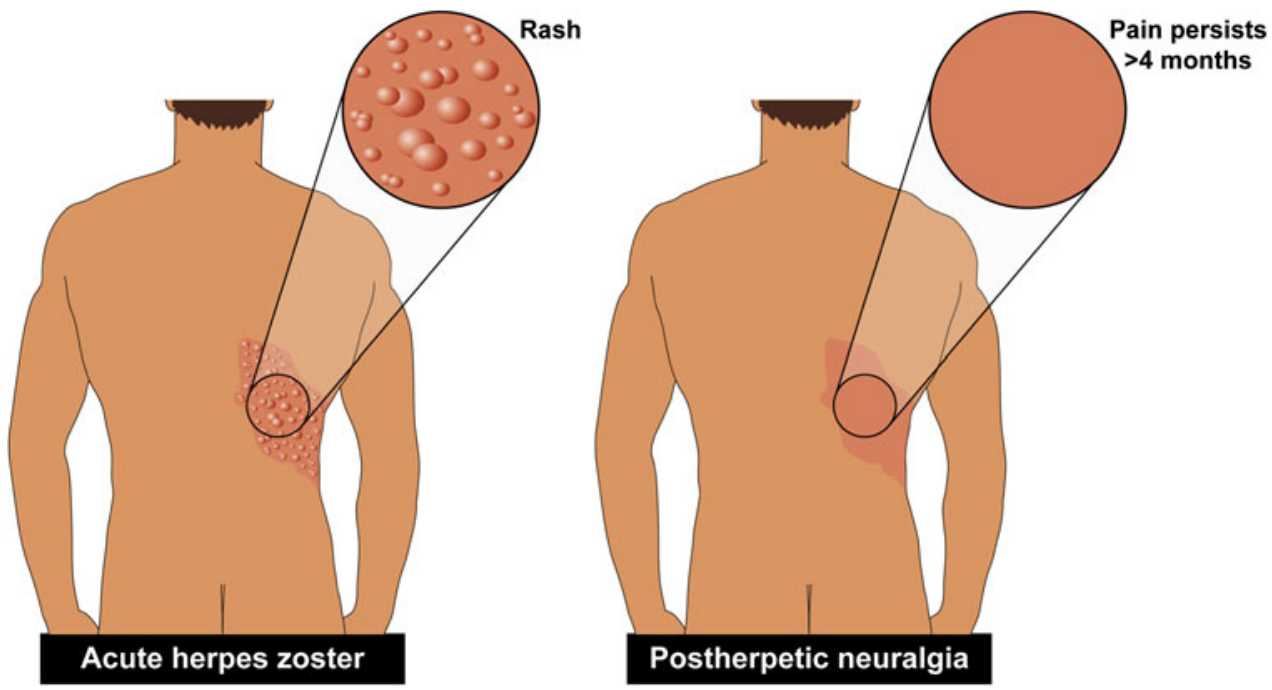
patients, most cases of herpes zoster and PHN emerge in older patients. The analysis revealed that the vaccine was most cost-effective for patients aged 70 years. Further, for all ages older than 60 years, the vaccine was more costeffective in women than in men, because women are disproportionately affected by herpes zoster and PHN compared with men.

It should be noted that the herpes zoster vaccine is a live virus vaccine and should not be administered to most immunocompromised patients, including patients with acquired or primary immunodeficiency, and must be administered with caution in patients receiving immunosuppressive drugs such as prednisone [12]. Immunocompromised patients represent nearly $10 \%$ of patients with AHZ [4]; hence, a substantial proportion of patients at risk for AHZ cannot be vaccinated.

Despite these caveats, the Centers for Disease Control and Prevention Advisory Committee on Immunization Practices now advocates the regular use of the herpes zoster vaccine in immunocompetent patients aged 60 years and older [12]. Nonetheless, current guidelines for the treatment of PHN, including guidelines issued in 2010 and 2011, emphasize pharmacological management. While some guidelines acknowledge the importance of prevention, none have highlighted preventative steps or recognized the role of the herpes zoster vaccine in preventing PHN [13-16]. The treatment of AHZ often includes antiviral agents such as aciclovir or famciclovir; however, these agents do not reduce the likelihood of subsequently developing PHN [17].

\section{Diagnosis of Postherpetic Neuralgia (PHN)}

\subsection{Patient History}

Although AHZ is typically easily recognizable, PHN can be difficult to diagnose because a patient with pain long after the AHZ rash has cleared up may not remember the rash or associate the current pain with it. In the diagnosis of PHN, routine questioning should attempt to identify the nature of the patient's pain (Table 1). In PHN, the pain is typically discretely localized and unilateral (i.e. dermatomal), intermittent, chronic and sufficiently intense to interfere with sleep and other normal daily activities. Additionally, the pain may have an itching, burning, sharp, stabbing or throbbing quality. Aggravating factors include light touch, such as the touch of clothing or standing in a shower. Relieving factors may include barriers to touch $[1,2]$.

The patient history may reveal conditions that may help differentially diagnosis PHN, including a recent history or the presence of herpes simplex virus, impetigo, candidiasis, contact dermatitis, insect bites, autoimmune blistering disease, dermatitis herpetiformis and drug-related eruptions [2].

With AHZ, however, nerve pain may emerge in the absence of any skin eruption, as in a condition called zoster sine herpete or in the presence of intercostal neuralgia [18, 19]. Nevertheless, pain following a documented episode of AHZ usually provides clear evidence for a diagnosis of PHN. This evidence may be particularly compelling when

Table 1 Diagnostic steps in postherpetic neuralgia

\begin{tabular}{|c|c|}
\hline Step & Diagnosis notes \\
\hline 1. Patient history & $\begin{array}{l}\text { Routine questioning should identify the source of the patient's pain } \\
\text { Pain is typically discrete and unilateral and displays an itching, burning, sharp, stabbing or throbbing quality } \\
\text { Pain is intermittent and chronic in nature } \\
\text { Pain is sufficiently intense to interfere with normal daily activities } \\
\text { Pain following a documented episode of AHZ provides compelling evidence for a diagnosis of PHN }\end{array}$ \\
\hline $\begin{array}{l}\text { 2. Physical } \\
\text { examination }\end{array}$ & $\begin{array}{l}\text { Areas of previous AHZ infection may manifest evidence of cutaneous scarring } \\
\text { Affected area may display either hypersensitivity or hyposensitivity to pain } \\
\text { Allodynia may occur in the pain-producing area } \\
\text { Autonomic changes may also occur in the affected area, including increased sweating }\end{array}$ \\
\hline $\begin{array}{l}\text { 3. Laboratory } \\
\text { investigations }\end{array}$ & $\begin{array}{l}\text { PHN diagnosis does not rely on laboratory evaluations } \\
\text { Viral culture or immunofluorescent staining may be used to distinguish herpes simplex from herpes zoster } \\
\text { Presence of antibodies to herpes zoster may help support diagnosis of subclinical herpes zoster infection, especially in } \\
\text { the case of zoster sine herpete } \\
\text { Other laboratory tests may be useful in confirming a herpes zoster infection, including immunoperoxidase staining, } \\
\text { histopathology and the Tzanck smear }\end{array}$ \\
\hline
\end{tabular}

$A H Z$ acute herpes zoster, $P H N$ postherpetic neuralgia 
additional PHN risk factors are present [2, 19], including advanced age, severe prodromal pain, severe rash, AHZ localized in the trigeminal dermatomes and brachial plexus, and symptoms of allodynia.

\subsection{Physical Examination}

During the physical examination, areas of previous AHZ may show evidence of cutaneous scarring [19]. In approximately $50 \%$ of patients with PHN, dynamic mechanical allodynia occurs in the pain-producing area in response to normally nonpainful stimuli, such as light touch by a brush. Thermal hyperalgesia is present in approximately one-third of patients. However, in some cases, decreased sensation or numbness may be experienced by the patient [20].

\section{Management of PHN}

\subsection{First-Line Therapies}

Tricyclic antidepressants, gabapentin and pregabalin, and the topical lidocaine $5 \%$ patch are each categorized as first-line treatments for PHN in guidelines issued by the American Academy of Neurology (2004) [14], the International Association for the Study of Pain (2007) [21], and the European Federation of Neurological Societies (2010) [13] (Table 2). However, topical lidocaine (as a gel, cream or patch) is considered a second-line or third-line therapy in British [16] and Canadian guidelines [22]. Opioids, tramadol, capsaicin cream and the capsaicin $8 \%$ patch are usually considered either second- or third-line treatments because of safety and/or efficacy disadvantages compared with first-line therapies.

\subsubsection{Tricyclic Antidepressants}

The tricyclic antidepressants amitriptyline, nortriptyline, imipramine and desipramine act by increasing serotonin

Table 2 Treatment options for postherpetic neuralgia

\begin{tabular}{|c|c|}
\hline Treatment type & Drug/drug class \\
\hline First-line & $\begin{array}{l}\text { Tricyclic antidepressants } \\
\text { Anticonvulsants such as gabapentin and pregabalin } \\
\text { Lidocaine } 5 \% \text { topical patch }\end{array}$ \\
\hline Second-line & $\begin{array}{l}\text { Opioid analgesics } \\
\text { Capsaicin } \\
\text { Tramadol }\end{array}$ \\
\hline $\begin{array}{l}\text { Combination } \\
\text { therapy }\end{array}$ & $\begin{array}{l}\text { Lidocaine } 5 \% \text { topical patch in combination with } \\
\text { systemic agent such as pregabalin } \\
\text { Systemic agents combinations such as gabapentin/ } \\
\text { nortriptyline or morphine/gabapentin }\end{array}$ \\
\hline
\end{tabular}

and norepinephrine levels and blocking voltage-dependent sodium channels and $\alpha$-adrenergic receptors, actions important in modulating descending pain pathways [23]. The efficacy of tricyclic antidepressants in the treatment of PHN pain is well established [3]. In a 2005 systematic review, the combined number needed to treat (NNT) with amitriptyline, nortriptyline or desipramine to obtain a single patient with a $50 \%$ reduction in PHN pain (compared with placebo) was 2.5 [24]. Despite their efficacy, tricyclic antidepressants for PHN may not be the most appropriate option for all patients. These agents display a relatively slow onset of action and have the potential for troublesome systemic adverse events, such as dry mouth, constipation, urinary retention and sedation. They have also been linked to cardiotoxic events, including myocardial infarction and cardiac dysrhythmias [2, 3, 25]. Thus, in some patientsespecially those most susceptible to tricyclic antidepressant adverse events and cardiotoxicities, such as the elderlyalternative PHN treatments may be considered.

\subsubsection{Gabapentin and Pregabalin}

The anticonvulsants gabapentin and pregabalin bind to $\alpha 2-\delta$ proteins, where they act as voltage-gated calcium channel blockers, inhibiting central pain pathways [23]. Although both of these agents act at the same receptor site, pregabalin may offer a more predictable and linear pharmacokinetic profile than gabapentin [2]. In the Cochrane Database of Systematic Reviews, the NNT to obtain a patient with a $50 \%$ reduction in pain was 7.5 for gabapentin [26] and 5.0-11 for pregabalin 300-600 $\mathrm{mg}$ [27]. As with tricyclic antidepressant treatment, adverse events with these agents may limit their use in some patients. In clinical studies, the most common adverse events with gabapentin and pregabalin included somnolence (up to $25 \%$ ) and dizziness (up to $46 \%$ ) [26, 27]. In addition, both agents may cause dry mouth, weight gain or peripheral oedema [28].

\subsubsection{Lidocaine $5 \%$ Patch}

Lidocaine blocks voltage-gated sodium channels, decreasing ectopic impulses generated by damaged primary afferent pain receptors, thus attenuating PHN pain perception [23]. The lidocaine $5 \%$ patch also incorporates a barrier effect similar to a cloth bandage. This therapy has a rapid onset of action ( $\leq 4 \mathrm{~h}$ ). In clinical trials (4-12 weeks), $\geq 50 \%$ reductions in pain have been reported in approximately one-quarter to onethird of patients [29-31]. Moreover, results from two 4-week open-label clinical studies indicated that the lidocaine $5 \%$ patch was more effective than pregabalin for alleviating PHN pain [29, 32]. Efficacy and safety for $\leq 24$ months has been demonstrated [33]. The NNT (calculated from small studies) for $\mathrm{a} \geq 50 \%$ reduction in neuropathic pain ranged from 2.0 to $4.4[22,24,34]$. 
The lidocaine $5 \%$ patch is generally well tolerated. The application of 3-4 patches daily results in systemic lidocaine concentrations of $<200 \mathrm{ng} / \mathrm{mL}$ [35, 36], a level much lower than that required to trigger systemic $(1,500 \mathrm{ng} / \mathrm{mL})$ adverse events or cardiovascular toxicity $(5,000 \mathrm{ng} / \mathrm{mL})$ [37]. In a 12-month open-label trial, [33] the most frequent treatment-related adverse events were mild-to-moderate application-site reactions, such as transient pruritus, erythema and dermatitis.

\subsection{Second-Line and Third-Line Therapies}

\subsubsection{Opioid Analgesics}

The role of opioid analgesics in the management of PHN remains controversial [2]. Strong opioids, such as oxycodone, morphine and methadone, provide effective analgesia in patients with PHN that is at least comparable to tricyclic antidepressants [38]. In a 2005 systematic review, the NNT for a $50 \%$ reduction in PHN pain with the strong opioids oxycodone and morphine was 2.5 and 2.7, respectively [24]. However, the long-term use of these analgesics presents a risk for potentially treatment-limiting adverse events, including nausea, sedation and constipation [38]. Tramadol, a centrally acting weak opioid and monoaminergic inhibitor, has proven less effective than strong opioids in PHN pain, but may be better tolerated than strong opioids [34]. The NNT for a $50 \%$ reduction in PHN pain with tramadol was 4.8 [24].

\subsubsection{Capsaicin}

Capsaicin, a vanilloid type I receptor agonist, provides analgesia by activating and, in turn, desensitizing nerve fibres in the skin over time [39]. Capsaicin cream and the capsaicin $8 \%$ patch have each demonstrated efficacy in patients with PHN [40-42]. Although capsaicin cream induces significant analgesia within 2 weeks of treatment initiation, maximal analgesic effect requires at least 4 weeks of treatment [42]. In a systematic review, the NNT (calculated from small studies) for a $50 \%$ reduction in PHN pain with capsaicin cream was 2.7-3.4 [24]. The capsaicin $8 \%$ patch has been shown to be more effective than conventional capsaicin cream formulations [43], though the NNT for a $50 \%$ reduction in PHN pain calculated using data from a large clinical trial was 10.4 [44].

With the use of capsaicin cream, patients should expect discomfort before the onset of meaningful pain relief. Capsaicin cream requires repeated applications before the onset of meaningful analgesia, and many patients are unable to tolerate the associated burning sensation [41, 42]. In fact, application-site reactions, such as burning, stinging and erythema, occurred in more than $60 \%$ of patients early in capsaicin treatment and were a major reason for treatment discontinuation [42]. The capsaicin $8 \%$ patch must be administered in a clinic or hospital under medical supervision as a single treatment every 3 months in combination with a local anaesthetic, a potentially major limitation to routine use. Even with a local anaesthetic, patients may experience post-procedural pain requiring systemic analgesics such as opioids [45].

\subsection{Rational Polypharmacy}

In the treatment of PHN and other neuropathic pain conditions, single-drug therapy may not provide sufficient analgesia even when administered at maximum tolerable doses [46]. A single agent alone may not be capable of effectively modifying the complex pain mechanisms that underlie PHN. Consequently, the use of multiple agents with different mechanisms of action may be required to provide enhanced pain relief. Toward this end, guidelines for the management of neuropathic pain advocate the use of combination regimens, especially the topical lidocaine $5 \%$ patch and an oral agent, when a single agent fails to offer acceptable PHN pain relief [21].

\subsubsection{Lidocaine $5 \%$ Patch Combination Therapy}

In a randomized, clinical trial, patients not responding sufficiently to the lidocaine $5 \%$ patch or pregabalin monotherapy after 4 weeks received combination therapy for 8 additional weeks [32]. During the monotherapy phase, 14 subjects $(29 \%)$ adequately responded to pregabalin and $25(50 \%)$ to the lidocaine $5 \%$ patch. During combination therapy, 18 patients added pregabalin to the lidocaine $5 \%$ patch, and 17 added the lidocaine $5 \%$ patch to pregabalin, at the same doses used in monotherapy. Combination therapy yielded clinically relevant reductions in PHN pain. During the monotherapy phase, the lidocaine $5 \%$ patch was significantly better tolerated than pregabalin, with the incidence of drug-related adverse events substantially lower in patients with the lidocaine $5 \%$ patch $(10 \%)$ than with pregabalin $(52.1 \%)$. Combination treatment was generally well tolerated. As with monotherapy, most adverse events were attributed to pregabalin treatment. A pregabalin dose-sparing effect was observed when the lidocaine $5 \%$ patch was added to pregabalin therapy. In a down-titration subtrial, pregabalin could be withdrawn from this combination therapy without pain relapse, implying that the improvement seen in pregabalin-treated patients with combination therapy may have been primarily the result of lidocaine treatment [32].

\subsubsection{Other Combination Therapies}

In the management of PHN pain, clinical studies also suggest that gabapentin combined with nortriptyline or 
morphine combined with gabapentin yields greater reductions in PHN pain than either modality alone; however, the rate of drug-specific adverse events remained high [3].

\section{Conclusions}

PHN remains a potentially debilitating and undertreated form of neuropathic pain. Over the past decade, clinical findings and treatment guidelines have underscored the importance of increased clinician awareness of the signs and symptoms of this chronically painful disorder and the importance of deploying evidence-based treatment modalities to improve outcome.

A medical consensus has emerged on what is considered best practice in the management of PHN. Because of the virtually universal presence of varicella zoster infection, a direct antecedent of AHZ, individuals at least 50 years of age should receive the $\mathrm{AHZ}$ vaccine to minimize their risk of both AHZ and PHN.

In patients with PHN, first-line systemic therapies, such as tricyclic antidepressants (amitriptyline, nortriptyline and desipramine) and gabapentinoids (gabapentin and pregabalin) have demonstrated efficacy, but may not be the most appropriate therapy for all patients because of their relatively slow onset of action and potential for treatmentlimiting systemic adverse events. The lidocaine $5 \%$ patch, listed as a first-line therapy in some guidelines, has a rapid onset of pain relief and a minimal risk for systemic adverse events, although application-site reactions occur in a minority of patients. Topical capsaicin cream or the capsaicin patch is considered second- or third-line therapy not only because capsaicin appears to be less effective than first-line therapies but also because it can trigger treatmentlimiting application-site reactions. Additionally, clinical studies reveal that, in the management of PHN, combination therapy with two agents that display different mechanisms of action may bolster efficacy in patients who do not adequately respond to either treatment alone. This strategy usually incorporates a systemic and topical therapy, chiefly the lidocaine $5 \%$ patch. Use of a topical analgesic in this way may provide additional analgesia without having a significant negative impact on the occurrence of systemic adverse events. In some patients, it may be clinically plausible to initiate the treatment of PHN with a topical agent, such as the lidocaine $5 \%$ patch, before resorting to a systemic therapy, and to switch to or add on a systemic therapy when necessary to achieve satisfactory pain control.

Acknowledgments Editorial support for this manuscript (literature search, document retrieval, medical writing and copyediting) was provided by Jeffrey Coleman, MA, James Kesslick, PhD, and Robert Gatley, MD, of Complete Healthcare Communications (Chadds Ford,
PA, USA), with funding from Endo Pharmaceuticals Inc. (Chadds Ford, PA, USA). The funding organization had no involvement in the literature search design, collection, management, analysis or interpretation of the literature reviewed in this manuscript and did not participate in the preparation, review or approval of the manuscript. A courtesy copy of the completed manuscript was provided to the funding organization at the time of its submission.

Conflict of interest Dr. Nalamachu has received honoraria as a speaker for Covidien, Lilly, Cephalon, Archimedes, Ipsen and ProStrakan; has been a paid consultant for Covidien, Endo, Insys, Nuvo, Ipsen, Cephalon, Zogenix and Endo; and has received research grants from Covidien, Endo, Zars, Nuvo, Insys and ProStrakan. Dr. MorleyForster has received honoraria as a speaker for Purdue Pharma Canada, has been a paid consultant for Janssen Ortho, and has received research grants from Pfizer Canada.

Open Access This article is distributed under the terms of the Creative Commons Attribution Noncommercial License which permits any noncommercial use, distribution, and reproduction in any medium, provided the original author(s) and the source are credited.

\section{References}

1. Johnson RW. Herpes zoster and postherpetic neuralgia. Expert Rev Vaccines. 2010;9(3 Suppl):21-6.

2. Sampathkumar P, Drage LA, Martin DP. Herpes zoster (shingles) and postherpetic neuralgia. Mayo Clin Proc. 2009;84(3):274-80.

3. Argoff CE. Review of current guidelines on the care of postherpetic neuralgia. Postgrad Med. 2011;123(5):134-42.

4. Yawn BP, Saddier P, Wollan PC, et al. A population-based study of the incidence and complication rates of herpes zoster before zoster vaccine introduction. Mayo Clin Proc. 2007;82(11): 1341-9.

5. Ragozzino MW, Melton LJ 3rd, Kurland LT, et al. Populationbased study of herpes zoster and its sequelae. Medicine (Baltimore). 1982;61(5):310-6.

6. Chen N, Li Q, Zhang Y, et al. Vaccination for preventing postherpetic neuralgia. Cochrane Database Syst Rev. 2011;3: CD007795

7. Zostavax ${ }^{\circledR}$ (zoster vaccine live): US prescribing information. Whitehouse Station, NJ: Merck \& Co., Inc., 2011.

8. Oxman MN, Levin MJ, Shingles Prevention Study Group. Vaccination against herpes zoster and postherpetic neuralgia. J Infect Dis. 2008;197(Suppl 2):S228-36.

9. Simberkoff MS, Arbeit RD, Johnson GR, et al. Safety of herpes zoster vaccine in the shingles prevention study: a randomized trial. Ann Intern Med. 2010;152(9):545-54.

10. Schmader KE, Levin MJ, Gnann JW Jr, et al. Efficacy, safety, and tolerability of herpes zoster vaccine in persons aged 50-59 years. Clin Infect Dis. 2012;54(7):922-8.

11. Rothberg MB, Virapongse A, Smith KJ. Cost-effectiveness of a vaccine to prevent herpes zoster and postherpetic neuralgia in older adults. Clin Infect Dis. 2007;44(10):1280-8.

12. Centers for Disease Control and Prevention. General recommendations on immunization: recommendations of the Advisory Committee on Immunization Practices (ACIP). MMWR Morb Mortal Wkly Rep. 2011;60(2):64.

13. Attal N, Cruccu G, Baron R, et al. EFNS guidelines on the pharmacological treatment of neuropathic pain: 2010 revision. Eur J Neurol. 2010;17(9):1113-e88.

14. Dubinsky RM, Kabbani H, El-Chami Z, et al. Practice parameter: treatment of postherpetic neuralgia: an evidence-based report of 
the Quality Standards Subcommittee of the American Academy of Neurology. Neurology. 2004;63(6):959-65.

15. Dworkin RH, Jensen MP, Gammaitoni AR, et al. Symptom profiles differ in patients with neuropathic versus non-neuropathic pain. J Pain. 2007;8(2):118-26.

16. Neuropathic pain: the pharmacological management of neuropathic pain in adults in non-specialist settings. London, UK: National Institute for Health and Clinical Excellence; 2011 February. Report No.: NICE clinical guideline 59.

17. Li Q, Chen N, Yang J, et al. Antiviral treatment for preventing postherpetic neuralgia. Cochrane Database Syst Rev 2009; (2):CD006866.

18. Chen SM, Chen JT, Kuan TS, et al. Myofascial trigger points in intercostal muscles secondary to herpes zoster infection of the intercostal nerve. Arch Phys Med Rehabil. 1998;79(3):336-8.

19. McElveen WA. Postherpetic neuralgia differential diagnoses. http://emedicine.medscape.com/article/1143066-overview. Accessed 11 May 2011.

20. Baron R, Tolle TR, Gockel U, et al. A cross-sectional cohort survey in 2100 patients with painful diabetic neuropathy and postherpetic neuralgia: differences in demographic data and sensory symptoms. Pain. 2009;146(1-2):34-40.

21. Dworkin RH, O'Connor AB, Backonja M, et al. Pharmacologic management of neuropathic pain: evidence-based recommendations. Pain. 2007;132(3):237-51.

22. Moulin DE, Clark AJ, Gilron I, et al. Pharmacological management of chronic neuropathic pain-consensus statement and guidelines from the Canadian Pain Society. Pain Res Manag. 2007;12(1):13-21.

23. Baron R. Neuropathic pain: a clinical perspective. Handb Exp Pharmacol. 2009;194:3-30.

24. Finnerup NB, Otto M, McQuay HJ, et al. Algorithm for neuropathic pain treatment: an evidence based proposal. Pain. 2005;118(3):289-305.

25. Saarto T, Wiffen PJ. Antidepressants for neuropathic pain: a Cochrane review. J Neurol Neurosurg Psychiatry. 2010;81(12):1372-3.

26. Moore RA, Wiffen PJ, Derry S, et al. Gabapentin for chronic neuropathic pain and fibromyalgia in adults. Cochrane Database Syst Rev 2011; (3):CD007938.

27. Moore RA, Straube S, Wiffen PJ, et al. Pregabalin for acute and chronic pain in adults. Cochrane Database Syst Rev 2009; (3):CD007076.

28. Tzellos TG, Toulis KA, Goulis DG, et al. Gabapentin and pregabalin in the treatment of fibromyalgia: a systematic review and a meta-analysis. J Clin Pharm Ther. 2010;35(6):639-56.

29. Baron R, Mayoral V, Leijon G, et al. $5 \%$ lidocaine medicated plaster versus pregabalin in post-herpetic neuralgia and diabetic polyneuropathy: an open-label, non-inferiority two-stage RCT study. Curr Med Res Opin. 2009;25(7):1663-76.

30. Baron R, Mayoral V, Leijon G, et al. Efficacy and safety of $5 \%$ lidocaine (lignocaine) medicated plaster in comparison with pregabalin in patients with postherpetic neuralgia and diabetic polyneuropathy: interim analysis from an open-label, two-stage adaptive, randomized, controlled trial. Clin Drug Investig. 2009;29(4):231-41.
31. Binder A, Bruxelle J, Rogers P, et al. Topical $5 \%$ lidocaine (lignocaine) medicated plaster treatment for post-herpetic neuralgia: results of a double-blind, placebo-controlled, multinational efficacy and safety trial. Clin Drug Investig. 2009;29(6):393-408.

32. Rehm S, Binder A, Baron R. Post-herpetic neuralgia: $5 \%$ lidocaine medicated plaster, pregabalin, or a combination of both? A randomized, open, clinical effectiveness study. Curr Med Res Opin. 2010;26(7):1607-19.

33. Hans G, Sabatowski R, Binder A, et al. Efficacy and tolerability of a $5 \%$ lidocaine medicated plaster for the topical treatment of post-herpetic neuralgia: results of a long-term study. Curr Med Res Opin. 2009;25(5):1295-305.

34. Hempenstall K, Nurmikko TJ, Johnson RW, et al. Analgesic therapy in postherpetic neuralgia: a quantitative systematic review. PLoS Med. 2005;2(7):e164.

35. Campbell BJ, Rowbotham M, Davies PS, et al. Systemic absorption of topical lidocaine in normal volunteers, patients with post-herpetic neuralgia, and patients with acute herpes zoster. J Pharm Sci. 2002;91(5):1343-50.

36. Gammaitoni AR, Davis MW. Pharmacokinetics and tolerability of lidocaine patch $5 \%$ with extended dosing. Ann Pharmacother. 2002;36(2):236-40.

37. Gammaitoni AR, Alvarez NA, Galer BS. Safety and tolerability of the lidocaine patch $5 \%$, a targeted peripheral analgesic: a review of the literature. J Clin Pharmacol. 2003;43(2):111-7.

38. Raja SN, Haythornthwaite JA, Pappagallo M, et al. Opioids versus antidepressants in postherpetic neuralgia: a randomized, placebo-controlled trial. Neurology. 2002;59(7):1015-21.

39. Nolano M, Simone DA, Wendelschafer-Crabb G, et al. Topical capsaicin in humans: parallel loss of epidermal nerve fibers and pain sensation. Pain. 1999;81(1-2):135-45.

40. Backonja MM, Malan TP, Vanhove GF, et al. NGX-4010, a highconcentration capsaicin patch, for the treatment of postherpetic neuralgia: a randomized, double-blind, controlled study with an open-label extension. Pain Med. 2010;11(4):600-8.

41. Bernstein JE, Korman NJ, Bickers DR, et al. Topical capsaicin treatment of chronic postherpetic neuralgia. J Am Acad Dermatol. 1989;21(2 Pt 1):265-70.

42. Watson CP, Tyler KL, Bickers DR, et al. A randomized vehiclecontrolled trial of topical capsaicin in the treatment of postherpetic neuralgia. Clin Ther. 1993;15(3):510-26.

43. Noto C, Pappagallo M, Szallasi A. NGX-4010, a high-concentration capsaicin dermal patch for lasting relief of peripheral neuropathic pain. Curr Opin Investig Drugs. 2009;10(7):702-10.

44. Irving GA, Backonja M, Rauck R, et al. NGX-4010, a capsaicin $8 \%$ dermal patch, administered alone or in combination with systemic neuropathic pain medications, reduces pain in patients with postherpetic neuralgia. Clin J Pain. 2012;28(2):101-7.

45. Qutenza ${ }^{\circledR}$ (capsaicin $8 \%$ patch): US prescribing information. San Mateo, CA: NeurogesX, Inc., 2009.

46. Vorobeychik Y, Gordin V, Mao J, et al. Combination therapy for neuropathic pain: a review of current evidence. CNS Drugs. 2011;25(12):1023-34. 